\title{
Methacrylation increase growth and differentiation of primary human osteoblasts for gelatin hydrogels
}

\author{
Mousumi Sukul $^{1} \cdot$ Giuseppe Cama $^{2,3} \cdot$ Peter Dubruel $^{2} \cdot$ Janne Elin Reseland $^{1} \cdot$ Håvard J. Haugen $^{1}$
}

Received: 22 January 2020 / Accepted: 15 April 2020 / Published online: 7 May 2020

(C) The Author(s) 2020

\begin{abstract}
The role of gelatin methacrylate hydrogels with varying degrees of methacrylation (69\% and $84 \%)$ was accessed with FTIR, NMR, microCT, and subsequent exposure to human osteoblasts. The cells responded positively to the degree of methacrylation and showed attachment, growth, and proliferated on both hydrogels. The cell reacted differently to the degree of methacrylation with higher proliferation on higher substitution; however, cell differentiation behavior was improved for less substitution. The secretion of late osteogenic markers (osteoprotegerin (OPG), osteopontin (OPN), and osteocalcin (OCN)) and angiogenic factor vascular endothelial growth factor (VEGF) was increased for gelatin methacrylate hydrogels with 69\% degree of methacrylation and thus would be the better candidate for future bone regenerative applications amongst the three tested hydrogels.
\end{abstract}

\section{Introduction}

Hydrogels, a specific class of hydrated polymers, are potential candidates for bone augmentation. Hydrogels can mimic the extracellular matrix of bone and integrate well with surrounding tissue, allowing a stabilized anchorage with host bone [1, 2]. They are degradable by endogenous enzymes or hydrolysis, which renders them the advantage of avoiding the complicacy of surgical removal of the implant and subsequent inflammation $[3,4]$. In the water-rich environment, hydrogels form a fibrous network, presenting the ability to entrap bioactive molecules and control the release as required to promote the healing process [5-7]. Flexible polymer chains of hydrogels enable them to be easily tailored to obtain required forms and shapes for implantation. Concentration of polymers, crosslinkers, and degree of crosslinking allows for the

\section{Håvard J. Haugen}

h.j.haugen@odont.uio.no

1 Department of Biomaterials, Institute of Clinical Dentistry, University of Oslo, Blindern, P.O. Box 1109, 0317 Oslo, Norway

2 Polymer Chemistry \& Biomaterials Group (PBM), Centre of Macromolecular Chemistry, Department of Organic and Macromolecular Chemistry, Ghent University, S4-Bis Krijgslaan 281, 9000 Ghent, Belgium

3 CAM Bioceramics B.V., Zernikedreef 6, 2333 CL Leiden, The Netherlands control over the physical properties of hydrogels such as pore size, porosity, rigidity, and degradation $[8,9]$.

Gelatin is a natural hydrophilic polymer well known for its use in different areas of tissue engineering including skin, neuron, cartilage, and bone [10-13]. Rising interesting in gelatin-based biomaterials is because of the fact that they are biocompatible, non-antigenic, and biodegradable $[14,15]$. Due to the presence of a large number of functional groups in the side chains, gelatin readily binds to chemical crosslinkers $[16,17]$. Bulcke et al. developed a methacrylic anhydride crosslinked gelatin hydrogel with controllable chemical networks [18]. Desirable compressive modulus and swelling properties of gelatin methacrylate (GM) can be obtained by controlling the polymer concentration, UV exposure time, and thermal gelation before UV exposure [19, 20]. Schuurman et al. reported high chondrocyte viability and the formation of cartilaginous tissue on GM hydrogels [20]. The porous architecture of GM hydrogel also provided a suitable microenvironment for neural stem cell survival and ingrowth [19]. Sustained release of BNP-2 from BNP-2 loaded GM hydrogels significantly enhanced the osteogenic differentiation of human adipose-derived stem cells compared with exogenous delivery of that growth factor [21]. Nguyen et al. incorporated GM microparticles with two different crosslinking densities into mouse embryonic stem cell aggregates and tested the effects on matrix metalloproteinase activity and cell differentiation [22].

In this study, we demonstrated the role of varying degrees of methacrylation of GM hydrogels to support the growth and differentiation of primary human osteoblasts. 


\section{Materials and methods}

\subsection{Synthesis of gelatin methacrylate hydrogels}

Gelatin type B (approximate isoelectric point of 5 and Bloom strength of 254) isolated from bovine skin by an alkaline treatment was obtained from Rousselot (Ghent, Belgium). Ethyl2-bromo propionate, sodium phosphate (dibasic, anhydrous, p.a.), potassium hydrogen phosphate, and $N$-vinylpyrrolidone (NVP, 99\%) were acquired from Acros (Geel, Belgium). Diethyl ether and chloroform were obtained from Chem-Lab (Zedelgem, Belgium) and 1,1,1,3,3-hexafluoro-2-propanol (HFP) from Fluorochem (Glossop, UK). The applied photoinitiator $0.8 \mathrm{w} / \mathrm{v} \%$ 1-[4-(2-hydroxyethoxy)-phenyl]-2hydroxy-2-methyl-1-propane-1-one (Irgacure® 2959) was obtained from BASF (Antwerp, Belgium). L-Lysine monohydrochloride was purchased from Merck (Darmstadt, Germany), dithiothreitol from Thermo Fisher Scientific (Waltham, USA), sodium azide (99\%), and iodine (I2) from Avocado Research Chemicals Ltd. (Karlsruhe, Germany) were prepared as reported [18, 23, 24]. The methacrylation was carried out as already described [25]. Samples were exposed to a $25-\mathrm{mW} / \mathrm{cm}^{2}$ UV-A light (LITE-Box G136 $365 \mathrm{~nm}$; NK-OPTIK, Germany) for $10 \mathrm{~min}$ to induce hydrogel crosslinking. The remaining chemicals were purchased from Sigma-Aldrich and used as received, unless stated otherwise.

\subsection{Pore size and porosity analysis}

The pore size and porosity of GM hydrogels were measured using a nano-computed tomograph (Multiscale $\mathrm{x}$-ray NanoCT, SkyScan 2211, Bruker microCT, Kontich, Belgium). Samples were prepared with a $1.5-\mathrm{mm}$ diameter and a 5-mm thickness. Scanned images were reconstructed using NRecon (SkyScan) and the porosity percentage was calculated using CTAn (SkyScan, Kontich, Belgium).

\subsection{Swelling}

In order to determine the material swelling properties, three disc-shaped samples $(d=1 \mathrm{~mm})$ for each sample type were freeze-dried and weighed (initial mass, $\mathrm{m} 0$ ). Subsequently, the samples were incubated for $24 \mathrm{~h}$ in deionized water at $37^{\circ} \mathrm{C}$. After swelling, the samples were again weighed (mt) and swelling degrees were calculated using the following equation:

Swelling $(\%)=\frac{W_{\mathrm{ht}}-W_{\mathrm{d} 0}}{W_{\mathrm{d} 0}} \times 100$

with $W_{\text {do }}=$ weight of dry gel at initial time 0 and $W_{\mathrm{ht}}=$ weight of hydrated gel at time $t$.
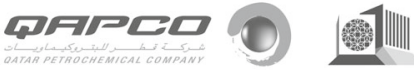

All these experiments were performed in duplicate. The results are reported as mean values with the corresponding SD.

\subsection{NMR}

The synthesized gelatin and alginate materials were structurally evaluated by conventional proton nuclear magnetic resonance $\left({ }^{1} \mathrm{H}-\mathrm{NMR}\right)$ spectroscopy according to previous experiments [26]. In brief, in order to apply conventional 1H-NMR spectroscopy, the dissolution of the polymer in a deuterated solvent is a prerequisite. In this work, the gelatin derivatives were dissolved in deuterated water $\left(\mathrm{D}_{2} \mathrm{O}, 99.90 \% \mathrm{D}\right)$ and spectra were recorded on a Bruker WH $500 \mathrm{MHz}$ at $40{ }^{\circ} \mathrm{C}$ with a zg30 pulse sequence, a spectral width of $10 \mathrm{kHz}$, an acquisition time of $3.28 \mathrm{~s}$, a preparation delay of $1 \mathrm{~s}$, and 16 accumulations. The chemical shift scale was calibrated with respect to tetramethylsilane (TMS) as the internal standard. Alginate derivatives on the other hand were first freeze-dried before dissolving in $\mathrm{D}_{2} \mathrm{O}(99.96 \%$ D). Resonance spectra were recorded at room temperature on an Agilent/Varian Inova 400 spectrometer using a 5-mm one NMR pulsedfield gradient (PFG) probe. The chemical shift scale was calibrated relatively to the trimethylsilyl resonance of sodium 2,2-dimethyl-2-silapentane-5-sulfonate (DSS) at $0 \mathrm{ppm}$. The signal of remaining water was suppressed by low power water presaturation. Spectra were acquired with a $90^{\circ}$ pulse of $6.3 \mu \mathrm{s}$, a spectral width of $6.5 \mathrm{kHz}$, an acquisition time of $2.6 \mathrm{~s}$, a preparation delay of $12 \mathrm{~s}$, and 128 accumulations. A line broadening of $1.0 \mathrm{~Hz}$ was applied prior to Fourier transformation to the frequency domain. Moreover, a highresolution magic angle spinning (HR-MAS) 1H-NMR spectrum was recorded on a Bruker Avance II 700 spectrometer (700.13 MHz) using a HR-MAS probe. Afterwards, the spectra were analyzed after baseline correction according to Van Vlierberghe et al. [26]

Infrared (IR) spectroscopy was applied for structural evaluation of the methacrylate derivative. For this purpose, a Frontier IR/NIR spectrometer from PerkinElmer was applied and measurement was performed in the mid-IR region ranging from 4000 to $400 \mathrm{~cm}^{-1}$. In addition, IR mapping was performed on the gelatin-alginate hydrogels. The surfaces of the dried hydrogel samples $(5 \times 5 \times 1 \mathrm{~mm})$ were scanned to evaluate the absorbance potentially occurring at the characteristic wavenumbers for gelatin and alginate. The IR mapping was performed using a Perkin Elmer Spectrum 100 FTIR spectrometer with a Spotlight 400 FTIR imaging system (PerkinElmer, Zaventem, Belgium).

\subsection{In vitro experiments}

Normal human osteoblast cells (NHOst) were used in the in vitro studies. Hydrogels were seeded with cells at a density 
of $3 \times 10^{4}$ cells $/ \mathrm{cm}^{2}$ and incubated in osteoblast growth media (Promocell, Heidelberg, Germany). The media was harvested at 1, 3, 7, 14, and 21 days. For confocal studies, cells cultured on the hydrogels were fixed at 7, 14, and 21 days and stained with fluorescent dyes. Each hydrogel group was tested in four parallels.

\subsection{Lactate dehydrogenase activity}

Cytotoxic effects of the hydrogels were tested by lactate dehydrogenase activity in the cell culture media using cytotoxicity detection kit (Roche Diagnostics, Indianapolis, IN, USA) according to producer's instructions. Cell culture media were harvested after 1 and 3 days of incubation. Duplicates of $50 \mu 1$ of the sample were added to $50 \mu \mathrm{l}$ of the mixture (catalyst and dye solution) and incubated in the dark for $30 \mathrm{~min}$ before measuring the absorbance in an absorbance microplate reader (ELx800, BioTek, VT, USA) at a wavelength of $490 \mathrm{~nm}$.

\subsection{Cell attachment}

Cell attachment and proliferation behavior on the hydrogels were studied after staining as previously described [27]. After 7 and 14 days of culture, the cell culture media were harvested, and the cells on the hydrogels were washed twice with PBS, fixed in 4\% paraformaldehyde for $10 \mathrm{~min}$, and washed with PBS. Fixed cells were permeabilized with $0.25 \%$ Triton$\mathrm{X}$ for $10 \mathrm{~min}$ and washed with PBS. A solution of $2.5 \%$ BSA (in PBS) was added to block non-specific binding. The cells were then stained with Alexa Fluor (568) conjugated phalloidin (Thermo Fischer Scientific, MA, USA, 1:400 working dilution in PBS) to visualize the actin filaments. Cell nuclei were stained with DAPI (Thermo Fischer Scientific, MA, USA, 1:1000 working dilution in PBS). Stained cells were observed under a confocal fluorescent microscope (Leica TCS SP8, Leica Microsystems, Wetzlar, Germany).

\subsection{Collagen expression}

To visualize the expression of collagen type 1 at day 21 , cells were stained according to the producer's instructions. Briefly, the cells were fixed and incubated with Anti-Collagen I antibody (COL-1) (Abcam, Cambridge, UK, 1:1000 working dilution in PBS) overnight at $4{ }^{\circ} \mathrm{C}$, and Alexa Fluor-488 conjugated Goat anti-mouse $\operatorname{IgG}(\mathrm{H}+\mathrm{L}$, Invitrogen, USA, 1:400 working dilution in PBS) for $1 \mathrm{~h}$ at room temperature. The Factin was labeled with Alexa Fluor (568) conjugated phalloidin and the nuclei were stained with DAPI. Stained cells were observed under a confocal fluorescent microscope.

\subsection{Alkaline phosphatase activity}

Alkaline phosphatase (ALP) activity was quantified by measuring the hydrolysis of $p$-nitrophenyl phosphate (pNPP, Invitrogen, Carlsbad, CA, USA) at $405 \mathrm{~nm}$. Standard curves using calf intestinal alkaline phosphatase (CIAP, Promega, Madison, WI, USA) were used as a reference. Aliquots of $25 \mu \mathrm{l}$ of samples together with $100 \mu \mathrm{l}$ of pNPP were incubated for $30 \mathrm{~min}$ at room temperature according to the protocol. The reaction was stopped with the addition of $50 \mu \mathrm{l}$ of $3 \mathrm{M} \mathrm{NaOH}$ and the absorbance was read in an absorbance microplate reader.

\subsection{Quantification of released bone markers and cytokines}

The effect of the hydrogels on the secretion of bone markers and cytokines in the culture media was measured using Milliplex Human Bone Panel kit (osteoprotegerin (OPG), osteopontin (OPN), osteocalcin (OC), sclerostin (SOST), Dickkopf-1 (DKK1)) and Human cytokine Panel kit (monocyte chemoattractant protein-1 (MCP-1), Interleukin 6 (IL-6), vascular endothelial growth factor (VEGF)) at 3, 7, 14, and 21 days of culture. All analyses were performed according to manufacturers' protocols. Multi-analyte profiling of the protein level in cell culture medium was performed on the Luminex 200 System using xMAP technology (Luminex, Austin, TX, USA). Acquired fluorescence data were analyzed by the XPONENT 3.1 software (Luminex). All data were normalized to cells grown on tissue culture plate.

\subsection{Statistical methods}

Statistical analysis was performed with SigmaPlot 14.0 (Systat Software Inc., Chicago, USA). Student $t$ test was used to evaluate the effect of different hydrogels compared with the control. The Mann-Whitney rank sum test was used if the results were not normally distributed. The significance level was set to $p \leq 0.05$.

\section{Results}

\subsection{Characterization of hydrogels}

Morphology of the porous G, GM69, and GM84 hydrogels was observed by NanoCT (Fig. 1). The pore sizes of G, GM69, and GM84 hydrogels was $94 \pm 46,229 \pm 105$, and $216 \pm 86 \mu \mathrm{m}$, respectively. All hydrogel groups consisted of interconnected pores with around $85 \%$ porosity. The strut thickness of $\mathrm{G}$ hydrogels was $13 \pm 7.0 \mu \mathrm{m}$. After methacrylamide crosslinking, the strut thickness of GM69

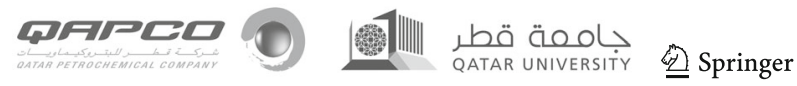



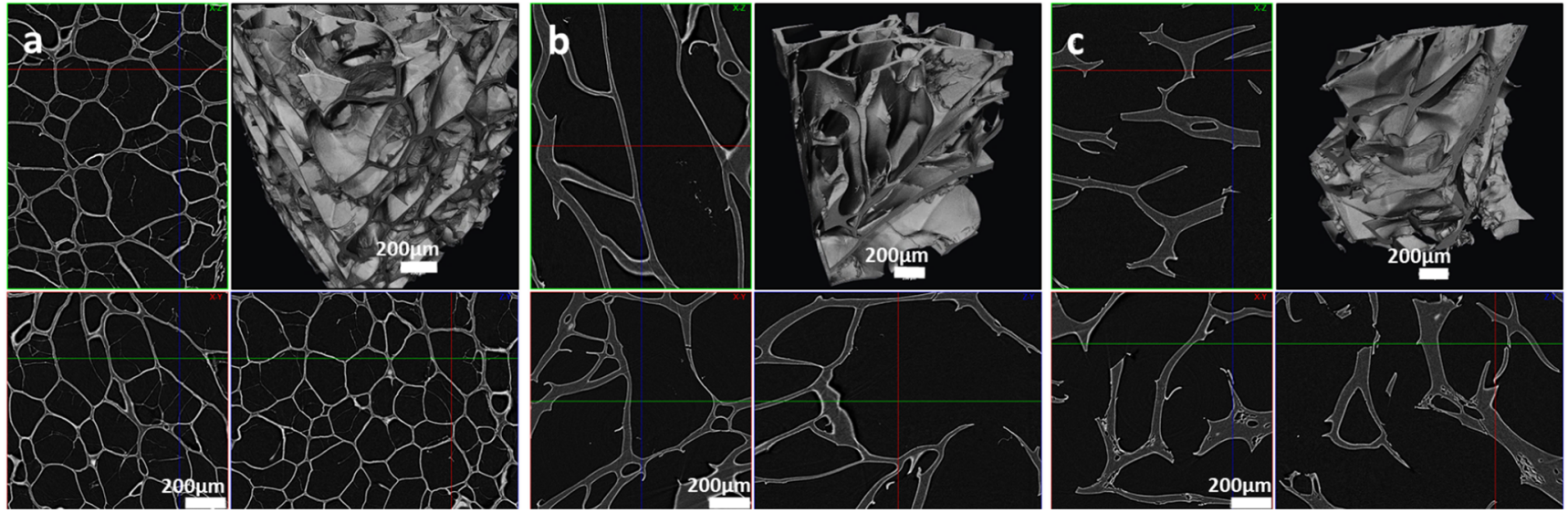

Fig. 1 MicroCT images of the different hydrogels used in the study where a cross-section of the $\mathrm{X}, \mathrm{Y}$, and ZX plan is displayed together with 3D images (upper corner). (a G, b GM69, c GM84)

and GM84 increased up to $41 \pm 16$ and $42 \pm 21 \mu \mathrm{m}$, respectively (Table 1).

The gels showed differentiation of swelling, where the highest swelling was observed for G (1684\% after $8 \mathrm{~h}$ and $1783 \%$ after $24 \mathrm{~h}$ ) and lowest for GM84 (938\% after $8 \mathrm{~h}$ and $956 \%$ after $24 \mathrm{~h}$ ). The swelling was linked to the degree of substitution (DS), where NMR showed the highest DS for GM84 (84\%) and lower for GM69 (69\%), whereas the degree of substitution G (Table 2). The FTIR analysis shows no difference between the different groups (Fig. 2).

\subsection{Cytotoxicity, cell attachment, and proliferation}

No cytotoxic effects on cultured NHOs were observed for all hydrogel groups compared with the control (tissue culture plate) both at days 1 and 3 (Fig. 3a).

Cells were cultured on the G, GM69, and GM84 hydrogels to determine if the degree of methacrylation affected the cell attachment and proliferation behavior. The morphology of the cells on the hydrogels was observed after 7 and 14 days of culture. More cells were attached to GM69 and GM84 hydrogels compared with $\mathrm{G}$ hydrogels after 7 days of culture. Attached cells on GM69 and GM84 hydrogels were elongated, with an increased number of extensions and filopodia. Cell numbers on GM69 hydrogels did not seem to increase notably at day 14 compared with that at day 7 , whereas the cells continued to proliferate on the surfaces of the GM84

Table 1 Pore morphology of gelatin methacrylate hydrogels $(n=3)$

\begin{tabular}{llcc}
\hline Samples & Porosity $(\%)$ & Pore size $(\mu \mathrm{m})$ & Strut thickness $(\mu \mathrm{m})$ \\
\hline G & $85 \pm 1.3$ & $48-140$ & $6-20$ \\
GM69 & $83 \pm 2.2$ & $124-334$ & $25-57$ \\
GM84 & $85 \pm 4.1$ & $130-302$ & $21-63$ \\
\hline
\end{tabular}

Data represent the mean \pm standard deviation hydrogels until day 14 which was evidenced by the intensity of dye bound to the actin filaments of dense multilayered cells (Fig 3b).

Cells cultured on the hydrogels were immune-stained with anti-collagen I antibody at day 21 to visualize collagen type 1 localization by confocal microscopy. Expressed collagen showed more intense labeling on the GM84 hydrogels compared with that on GM69 hydrogels (Fig. 3c).

\subsection{Bone markers and cytokines secreted in the culture medium}

GM69 and GM84 hydrogels induced 160\% and 190\% $(p<0.05)$ increase, respectively, in the ALP activity at day 3 compared with the control. The activity was then decreased down to control. G hydrogels caused an $80 \%$ increase at day 3 and a $140 \%$ increase at day $7(p<0.01)$ compared with the control. Then, the secretion was downregulated (Fig. 4a).

Secretion of OPN in the media was lower compared with the control at day 3 for GM69 hydrogels $(p<0.05)$, which later increased slightly up to 14 days and became stable afterwards. Secretion of OPN was lower compared with the control

Table 2 Characterization of gelatin methacrylate hydrogels $(n=4)$

\begin{tabular}{|c|c|c|c|c|c|}
\hline Acronym & $\begin{array}{l}\text { Swelling at } \\
8 \mathrm{~h}(\%), \\
\mathrm{Av} \pm \\
\text { St.Dev }\end{array}$ & $\begin{array}{l}\text { Swelling at } \\
24(\%), \\
\text { Av } \pm \text { St.Dev }\end{array}$ & $\begin{array}{l}\text { Increase of wt. } \\
\text { at } 24 \mathrm{~h} \text { post } \\
\text { immersion in } \\
\text { MQ water, Av } \\
\pm \text { St.Dev }\end{array}$ & $\begin{array}{l}\text { Gel } \\
\text { fraction } \\
(\%), \text { Av } \\
\pm \\
\text { St.Dev }\end{array}$ & $\begin{array}{l}\mathrm{DS} \\
(\%) \\
{ }^{1} \mathrm{H}- \\
\mathrm{NMR} \\
(\%)\end{array}$ \\
\hline G & $\begin{array}{l}1684 \pm 46 \\
\mathrm{pH}=6.9\end{array}$ & $\begin{array}{l}1783 \pm 51 \\
\mathrm{pH}=7.1\end{array}$ & $6 \pm 4.3$ & $71 \pm 7$ & 13 \\
\hline GM69 & $\begin{array}{l}1249 \pm 186 \\
\mathrm{pH}=6.9\end{array}$ & $\begin{array}{l}1667 \pm 214 \\
\mathrm{pH}=7.1\end{array}$ & $3.4 \pm 3.7$ & $68 \pm 9$ & 69 \\
\hline GM84 & $\begin{array}{l}938 \pm 38 \\
\mathrm{pH}=7\end{array}$ & $\begin{array}{l}956 \pm 32 \\
\mathrm{pH}=7.04\end{array}$ & $1.9 \pm 0.8$ & $87 \pm 2$ & 84 \\
\hline
\end{tabular}

$D S$ degree of substitution, $A v \pm S t . D e v$ average value \pm standard deviation 
Fig. 2 FITR profile of the different gels

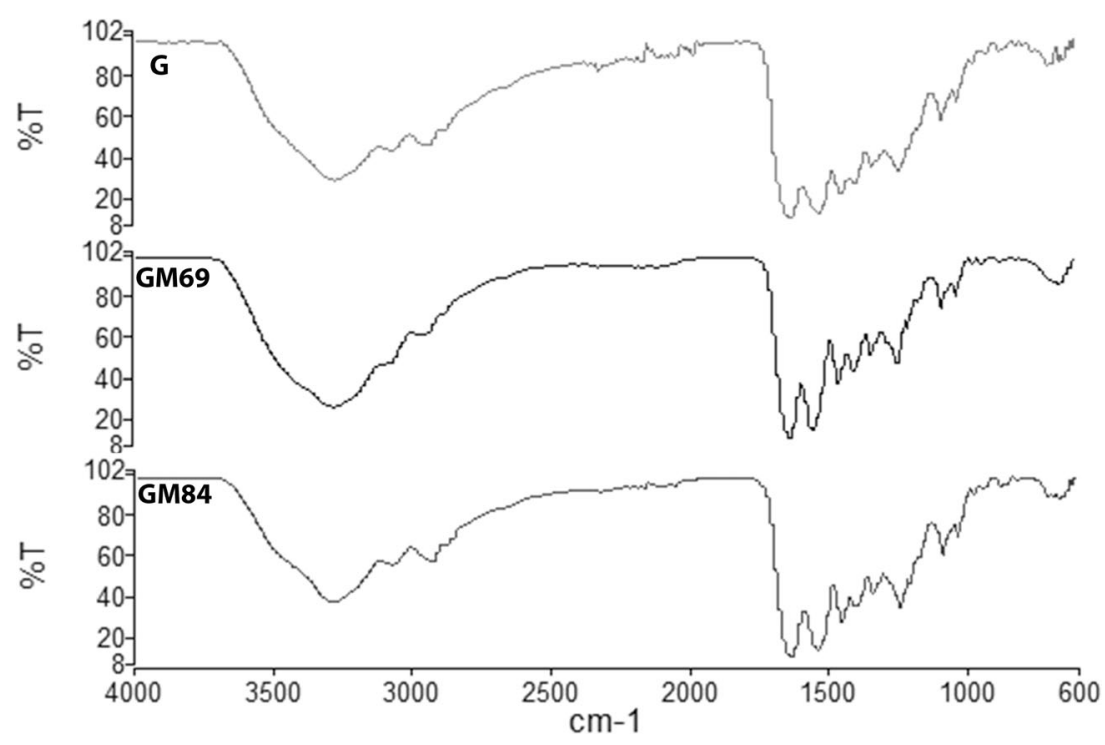

at all time points for GM84 hydrogels. At day 14, the secretion was significantly upregulated by GM69 hydrogels compared with GM84 hydrogels $(p<0.05)$. G hydrogels did not influence OPN secretion significantly at any time points when compared with the control (Fig. 4b).
At day 3, the secretion of OCN was not significantly different compared with the control for any hydrogel groups. After this time point, GM84 and G hydrogels reduced the section significantly compared with the control $(p<0.05)$, whereas GM69 hydrogels increased OCN

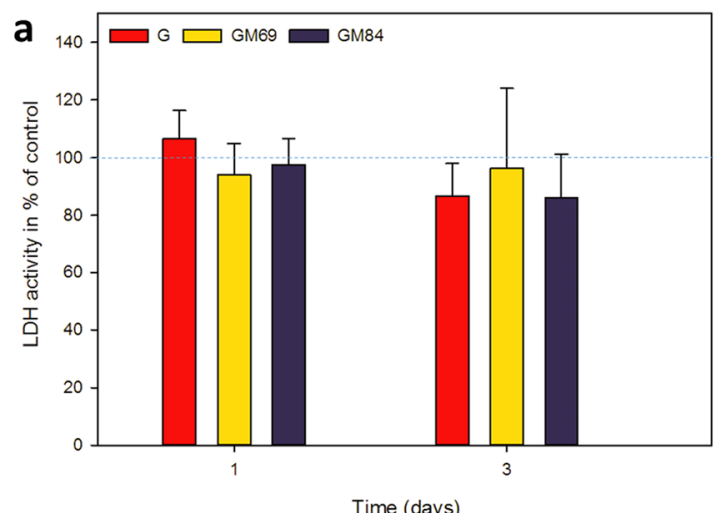

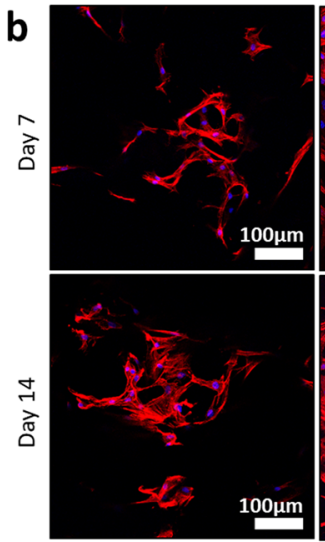

G
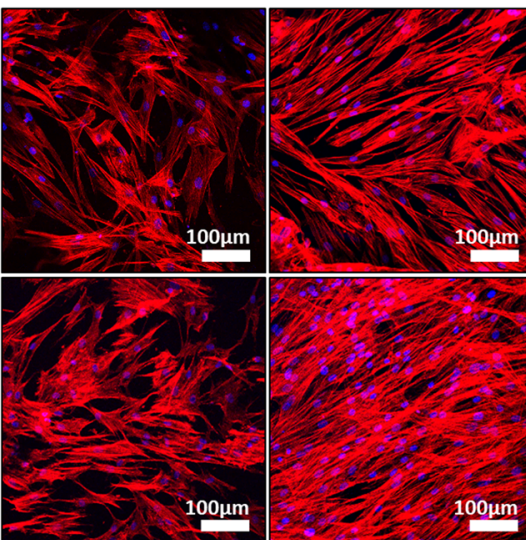

GM69

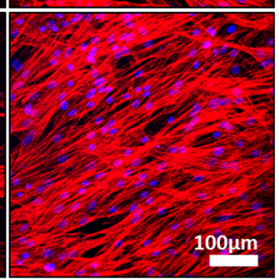

GM84

Fig. 3 LDH showing no cytotoxic effect of the hydrogels and no significant difference between the groups (a). Confocal images after 7 and 14 days of cultured primary human osteoblast stained with collagen-1 and F-actin +collagen 1. Cells continued to proliferate on the surfaces of

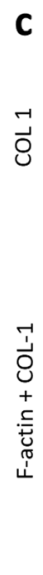

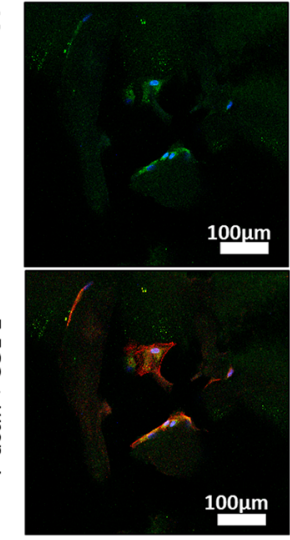

G

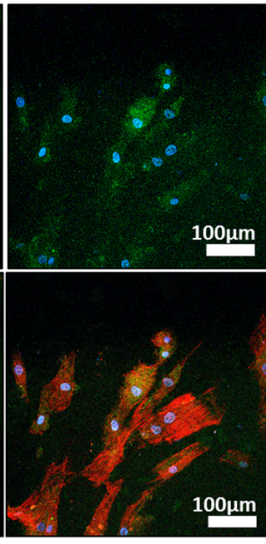

GM69

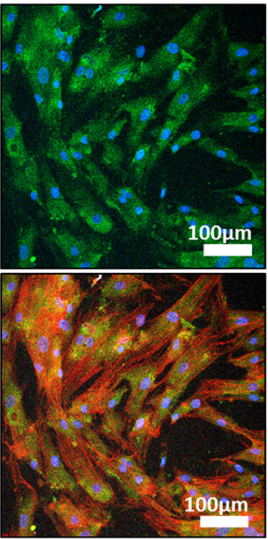

GM84 the GM84 hydrogels until day 14, which was evidenced by the intensity of dye bound to the actin filaments of dense multilayered cells (b). Expressed collagen showed more intense labeling on the GM84 hydrogels compared with that on GM69 hydrogels $(\mathbf{c})(n=4)$ 

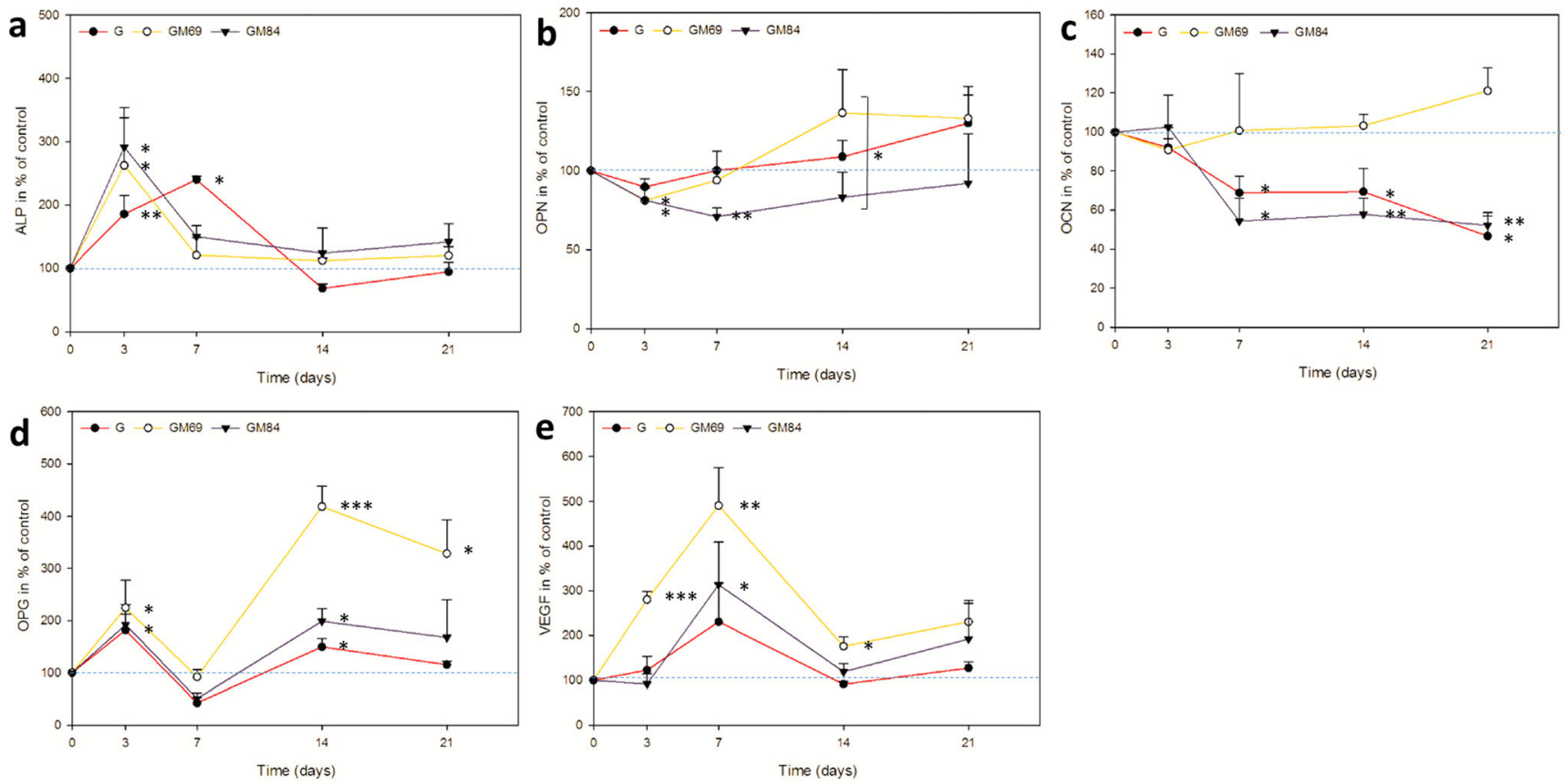

Fig. 4 Cellular response to the hydrogels measured by Milliplex Human Bone Panel kit where secretion of various bone markers and cytokines in the culture media was measured for cultivation up to 21 days. a Alkaline phosphatase (ALP), b osteopontin (OPN), c osteocalcin (OCN), d

osteoprotegerin (OPG), e vascular endothelial growth factor (VEGF). The response has been normalized to cell culture tissue plate. ${ }^{*} p<0.05$ versus control, $* * p<0.05$ versus $\mathrm{G}$ and GM69, $* * p<0.05$ versus GM69 $(n=4)$

secretion slightly at day 21 when compared with the control (Fig. 4c).

OPG secretion was initially enhanced by GM69 and GM84 hydrogels up to $120 \%$ and $90 \%$, respectively $(p<0.05)$, at day 3 compared with the control and downregulated at day 7 . At day 14, the secretion reached a $320 \%$ increase by GM69 $(p<0.001)$ and only a 100\% increase by GM84 hydrogels $(p<0.05)$ compared with the control. Then, the secretion was downregulated slightly by both hydrogels. Secretion was not significantly changed by $\mathrm{G}$ hydrogels compared with the control at any time points except at day 14, where the secretion was increased up to 50\% (Fig. 4d).

The secretion of VEGF was enhanced by GM69 hydrogels to $180 \%(p<0.001)$ at day 3 which reached a $390 \%(p<0.01)$ increase at day 7 compared with the control, then decreased. The secretion was not influenced by GM84 hydrogels at day 3 but increased to $210 \%(p<0.05)$ at day 7 and downregulated afterwards. Secretion induced by $\mathrm{G}$ did not reach a statistical significance at any time points when compared with the control (Fig. 4e).

\section{Discussion}

In this study, we demonstrated the role of gelatin methacrylate (GM) hydrogels with varying degrees of methacrylation to support the growth and differentiation of primary human osteoblasts. We found that cell behavior was dependent on the degree of methacrylation. Highly methacrylated hydrogels (GM84) were being more permissive to cell proliferation than the softer ones (GM69) for a longer period, whereas the softer hydrogels favored the secretion of bone markers and cytokines after a certain point when the cell proliferation was reduced.

The photocrosslinkable gelatin methacrylate hydrogels were synthesized by adding methacrylate groups to the amine group containing side chains of gelatin. We observed that the pore size and strut thickness of the hydrogels can be tuned by altering the degree of methacrylation. Chen et al. demonstrated that the porosity and the degradability could be controlled by modifying the methacrylation degree to achieve a desirable mechanical stiffness without compromising the cytocompatibility [28]. In our study, both GM hydrogels supported cell attachment and proliferation when seeded on its surface. Having gelatin as the backbone, GM hydrogels provided appropriate cell binding motifs, which promoted cell adhesion and proliferation [29]. The reason for the higher number of cells attached to GM84 hydrogels might be the degree of stiffness of the hydrogel. Cell proliferation increases with the stiffness of the matrix. Cells on the stiffer matrix generally exhibit more organized cytoskeletons and more stable focal adhesions [30]. Storage modulus has been reported to be $18 \pm 1.3 \mathrm{kPa}$ for UV cured $10 \mathrm{w} / \mathrm{v} \%$ gel-MOD [31]. Nichol et al. demonstrated an increase in stiffness for a higher degree of methacrylation increased, where $81 \%$ DMA responded to $18 \mathrm{kPa}$ and $54 \%$ responded to $10 \mathrm{kPa}$ in 
compressive modulus [29]. The modulation of differentiation pathways by hydrogel stiffness was largely independent of the degree of cell proliferation. The effect of different stiffness properties of Gel-MOD on osteo- and chondrogenic differentiation has been investigated with encapsulated hASC/hTERT microspheroids. Žigon-Branc et al. found out that regardless of the hydrogel stiffness (for the samples stimulated with osteogenic medium), the expression of selected markers RUNX2, BGLAP, ALPL, and COL1A1 was not conclusive. However, when microspheroids were placed in softer GelMOD, these started to outgrow and interconnect within a few days, and their protrusion was slower or more limited in stiffer Gel-MOD hydrogels. [32]. Previous studies have shown a significant influence on stem cell migration, proliferation, and differentiation by varying stiffness of twodimensional (2D) or 3D substrates [33]. The expression level of ALP, the early bone osteogenic marker, was similar on both hydrogels. However, the late bone markers such as OPN, $\mathrm{OCN}$, and OPG became the predominant osteogenic message for the softer gels at later time points. A much higher expression of VEGF was observed for the softer gel when compared with the stiffer gel. Although there is a positive correlation between the increasing matrix stiffness and the increasing cell differentiation, the stiffness optimum for differentiation varies significantly from cell to cell. Neurons prefer to grow well and form multiple branches on softer gels while glial cells do not survive well on these deformable matrices [34]. Myoblasts are able to form myotubes on materials of varied elasticity. However, myotube striations only emerge on gels with stiffness similar to normal muscle [35]. Human umbilical vein endothelial cells (HUVEC) form multi-cellular structures with larger lumens having spread appearance on stiff gels but thin, multi-cellular structures with small lumens on softer gels [36]. Neutrophils, on the other hand, are insensitive to stiffness changes and spread equally well on matrices with a very wide range of elasticity [37].

\section{Conclusion}

In summary, we tested the role of gelatin methacrylate hydrogels with varying degrees of methacrylation $(69 \%$ and $84 \%)$ to support the growth and differentiation of primary human osteoblasts. Cells grew and proliferated on both methacrylated hydrogels but the degree of proliferation was a higher degree of methacrylation. Conversely, cell differentiation behavior was improved on less degree of substitution. Although the expression of early osteogenic marker (ALP) did not seem to be influenced by the DS of the hydrogels, the secretion of late osteogenic markers (OPN, OCN, and OPG) and angiogenic factor (VEGF) was increased by less degree of methacrylation. Based on these data, gelatin methacrylate hydrogels with a $69 \%$ degree of methacrylation can be proposed in future bone regenerative applications.

Acknowledgments The authors would like to thank Aina-Mari Lian and Liebert Parreiras Nogueira for the contribution in Luminex experiment and NanoCT.

Funding information Open Access funding provided by University of Oslo (incl Oslo University Hospital). This research was supported by "ERA-NET: Mar. Origin Biopolymers as Inno. Building Blocks from the Sea for the Development of bioresorbable Multilayered Membranes" with acronym Blueteeth "funded by Research Council of Norwegian (RCN) grant number 269522 and Marine Biotechnology ERA-NET (ERA-MarineBiotech)." The study was performed in compliance with national and european ethical standards for the use of human cells.

\section{Compliance with ethical standards}

Conflict of interest The authors declare that they have no conflict of interest.

Open Access This article is licensed under a Creative Commons Attribution 4.0 International License, which permits use, sharing, adaptation, distribution and reproduction in any medium or format, as long as you give appropriate credit to the original author(s) and the source, provide a link to the Creative Commons licence, and indicate if changes were made. The images or other third party material in this article are included in the article's Creative Commons licence, unless indicated otherwise in a credit line to the material. If material is not included in the article's Creative Commons licence and your intended use is not permitted by statutory regulation or exceeds the permitted use, you will need to obtain permission directly from the copyright holder. To view a copy of this licence, visit http://creativecommons.org/licenses/by/4.0/.

\section{References}

1. Wang D-A, Varghese S, Sharma B, et al. Multifunctional chondroitin sulphate for cartilage tissue-biomaterial integration [Article]. Nature Materials. 2007 04/15/online;6:385. https://doi.org/10. 1038/nmat1890 https://www.nature.com/articles/nmat1890\# supplementary-information

2. D.S. Thoma, C.C. Villar, D.L. Cochran, C.H.F. Hämmerle, R.E. Jung, Tissue integration of collagen-based matrices: an experimental study in mice. Clin. Oral Implants Res. 23(12), 1333-1339 (2012). https://doi.org/10.1111/j.1600-0501.2011.02356.x

3. Y. Li, J. Rodrigues, H. Tomas, Injectable and biodegradable hydrogels: gelation, biodegradation and biomedical applications. Chem. Soc. Rev. 41(6), 2193-2221 (2012)

4. J.M. Knipe, F. Chen, N.A. Peppas, Enzymatic biodegradation of hydrogels for protein delivery targeted to the small intestine. Biomacromolecules. 16(3), 962-972 (2015)

5. S. Young, M. Wong, Y. Tabata, et al., Gelatin as a delivery vehicle for the controlled release of bioactive molecules. J Control Release 109(1), 256-274 (2005). https://doi.org/10.1016/j.jconrel.2005.09. 023

6. S. Saber-Samandari, S. Saber-Samandari, Biocompatible nanocomposite scaffolds based on copolymer-grafted chitosan for bone tissue engineering with drug delivery capability. Mater Sci Eng C 75, 721-732 (2017). https://doi.org/10.1016/j.msec.2017.02.112 
7. B. An, Y.-S. Lin, B. Brodsky, Collagen interactions: drug design and delivery. Adv Drug Deliv Rev 97, 69-84 (2016). https://doi. org/10.1016/j.addr.2015.11.013

8. W.E. Hennink, C.F. van Nostrum, Novel crosslinking methods to design hydrogels. Adv Drug Deliv Rev 64, 223-236 (2012). https:// doi.org/10.1016/j.addr.2012.09.009

9. H. Shih, C.-C. Lin, Cross-linking and degradation of step-growth hydrogels formed by thiol-ene photoclick chemistry. Biomacromolecules 13(7), 2003-2012 (2012). https://doi.org/10. 1021/bm300752j

10. B.S. Kwak, W. Choi, J.-W. Jeon, et al., In vitro 3D skin model using gelatin methacrylate hydrogel. J Ind Eng Chem 66, 254-261 (2018). https://doi.org/10.1016/j.jiec.2018.05.037

11. H.-W. Han, Y.-T. Hou, S.-H. Hsu, Angiogenic potential of cospheroids of neural stem cells and endothelial cells in injectable gelatin-based hydrogel. Mater Sci Eng C 99, 140-149 (2019). https://doi.org/10.1016/j.msec.2019.01.089

12. A.K. Kudva, A.D. Dikina, F.P. Luyten, et al., Gelatin microspheres releasing transforming growth factor drive in vitro chondrogenesis of human periosteum derived cells in micromass culture. Acta Biomater 90, 287-299 (2019). https://doi.org/10.1016/j.actbio. 2019.03.039

13. M. Yamamoto, A. Hokugo, Y. Takahashi, et al., Combination of BMP-2-releasing gelatin/ $\beta$-TCP sponges with autologous bone marrow for bone regeneration of X-ray-irradiated rabbit ulnar defects. Biomaterials 56, 18-25 (2015). https://doi.org/10.1016/j. biomaterials.2015.03.057

14. S.J. Lee, J.Y. Yhee, S.H. Kim, et al., Biocompatible gelatin nanoparticles for tumor-targeted delivery of polymerized siRNA in tumor-bearing mice. J Control Release 172(1), 358-366 (2013). https://doi.org/10.1016/j.jconrel.2013.09.002

15. Y.-S. Chen, J.-Y. Chang, C.-Y. Cheng, et al., An in vivo evaluation of a biodegradable genipin-cross-linked gelatin peripheral nerve guide conduit material. Biomaterials 26(18), 3911-3918 (2005). https://doi.org/10.1016/j.biomaterials.2004.09.060

16. A. Bigi, G. Cojazzi, S. Panzavolta, et al., Mechanical and thermal properties of gelatin films at different degrees of glutaraldehyde crosslinking. Biomaterials 22(8), 763-768 (2001). https://doi.org/ 10.1016/S0142-9612(00)00236-2

17. A. Bigi, G. Cojazzi, S. Panzavolta, et al., Stabilization of gelatin films by crosslinking with genipin. Biomaterials 23(24), 48274832 (2002). https://doi.org/10.1016/S0142-9612(02)00235-1

18. A.I. Van Den Bulcke, B. Bogdanov, N. De Rooze, et al., Structural and rheological properties of methacrylamide modified gelatin hydrogels. Biomacromolecules. 1(1), 31-38 (2000). https://doi. org $/ 10.1021 / \mathrm{bm} 990017 \mathrm{~d}$

19. Zhu W, Harris BT, Zhang LG, editors. Gelatin methacrylamide hydrogel with graphene nanoplatelets for neural cell-laden 3D bioprinting. 2016 38th Annual International Conference of the IEEE Engineering in Medicine and Biology Society (EMBC); 2016 16-20 Aug. 2016

20. W. Schuurman, P.A. Levett, M.W. Pot, P.R. van Weeren, W.J.A. Dhert, D.W. Hutmacher, F.P.W. Melchels, T.J. Klein, J. Malda, Gelatin-methacrylamide hydrogels as potential biomaterials for fabrication of tissue-engineered cartilage constructs. Macromol. Biosci. 13(5), 551-561 (2013). https://doi.org/10.1002/mabi. 201200471

21. J.E. Samorezov, E.B. Headley, C.R. Everett, E. Alsberg, Sustained presentation of BMP-2 enhances osteogenic differentiation of human adipose-derived stem cells in gelatin hydrogels. J. Biomed. Mater. Res. A 104(6), 1387-1397 (2016). https://doi.org/10.1002/ jbm.a.35668
22. A.H. Nguyen, Y. Wang, D.E. White, et al., MMP-mediated mesenchymal morphogenesis of pluripotent stem cell aggregates stimulated by gelatin methacrylate microparticle incorporation. Biomaterials 76, 66-75 (2016). https://doi.org/10.1016/j. biomaterials.2015.10.043

23. S. Benedikt, J. Wang, M. Markovic, N. Moszner, K. Dietliker, A. Ovsianikov, H. Grützmacher, R. Liska, Highly efficient watersoluble visible light photoinitiators. J Polym Sci Pol Chem. 54(4), 473-479 (2016). https://doi.org/10.1002/pola.27903

24. A. Ovsianikov, A. Deiwick, S. Van Vlierberghe, et al., Laser fabrication of three-dimensional CAD scaffolds from photosensitive gelatin for applications in tissue engineering. Biomacromolecules. 12(4), 851-858 (2011). https://doi.org/10.1021/bm1015305

25. Mandt D, Gruber P, Markovic M, et al. Fabrication of biomimetic placental barrier structures within a microfluidic device utilizing two-photon polymerization. Int J Bioprinting. 2018;4(2). UNSP 144 https://doi.org/10.18063/IJB.v4i2.144

26. S. Van Vlierberghe, B. Fritzinger, J.C. Martins, et al., Hydrogel network formation revised: high-resolution magic angle spinning nuclear magnetic resonance as a powerful tool for measuring absolute hydrogel cross-link efficiencies. Appl. Spectrosc. 64(10), 1176-1180 (2010 Oct). https://doi.org/10.1366/ 000370210792973550

27. B.-R. Kim, T.B.L. Nguyen, Y.-K. Min, B.T. Lee, In vitro and in vivo studies of BMP-2-loaded PCL-gelatin-BCP electrospun scaffolds. Tissue Eng. A 20(23-24), 3279-3289 (2014)

28. Y.C. Chen, R.Z. Lin, H. Qi, Y. Yang, H. Bae, J.M. Melero-Martin, A. Khademhosseini, Functional human vascular network generated in photocrosslinkable gelatin methacrylate hydrogels. Adv. Funct. Mater. 22(10), 2027-2039 (2012)

29. J.W. Nichol, S.T. Koshy, H. Bae, et al., Cell-laden microengineered gelatin methacrylate hydrogels. Biomaterials 31(21), 5536-5544 (2010). https://doi.org/10.1016/j.biomaterials.2010.03.064

30. D.E. Discher, P. Janmey, Y.-L. Wang, Tissue cells feel and respond to the stiffness of their substrate. Science. 310(5751), 1139-1143 (2005)

31. J. Van Hoorick, P. Gruber, M. Markovic, et al., Cross-linkable gelatins with superior mechanical properties through carboxylic acid modification: increasing the two-photon polymerization potential. Biomacromolecules. 18(10), 3260-3272 (2017). https://doi.org/10. 1021/acs.biomac.7b00905

32. S. Zigon-Branc, M. Markovic, J. Van Hoorick, et al., Impact of hydrogel stiffness on differentiation of human adipose-derived stem cell microspheroids. Tissue Eng. A 25(19-20), 1369-1380 (2019). https://doi.org/10.1089/ten.TEA.2018.0237

33. A. Higuchi, Q.D. Ling, Y. Chang, S.T. Hsu, A. Umezawa, Physical cues of biomaterials guide stem cell differentiation fate. Chem. Rev. 113(5), 3297-3328 (2013). https://doi.org/10.1021/cr300426x

34. L.A. Flanagan, Y.-E. Ju, B. Marg, M. Osterfield, P.A. Janmey, Neurite branching on deformable substrates. Neuroreport. 13(18), 2411-2415 (2002)

35. A.J. Engler, M.A. Griffin, S. Sen, C.G. Bönnemann, H.L. Sweeney, D.E. Discher, Myotubes differentiate optimally on substrates with tissue-like stiffness: pathological implications for soft or stiff microenvironments. J. Cell Biol. 166(6), 877-887 (2004)

36. A.L. Sieminski, R.P. Hebbel, K.J. Gooch, The relative magnitudes of endothelial force generation and matrix stiffness modulate capillary morphogenesis in vitro. Exp Cell Res 297(2), 574-584 (2004). https://doi.org/10.1016/j.yexcr.2004.03.035

37. T. Yeung, P.C. Georges, L.A. Flanagan, B. Marg, M. Ortiz, M. Funaki, N. Zahir, W. Ming, V. Weaver, P.A. Janmey, Effects of substrate stiffness on cell morphology, cytoskeletal structure, and adhesion. Cell Motil. Cytoskeleton 60(1), 24-34 (2005) 